\title{
The Importance of Computed Tomography Findings in Detecting Tuberculous Addison's Disease
}

\author{
Sara Ferreira, Margarida Freitas-Silva \\ Serviço de Medicina Interna, Centro Hospitalar de S. João, Porto, Portugal
}

Received: $14 / 04 / 2017$

Accepted: 07/06/2017

Published: 20/06/2017

How to cite this article: Ferreira S, Freitas-Silva M. The importance of computed tomography findings in detection tuberculous Addison's disease. EJCRIM 2017;4: doi:10.12890/2017_000622

Conflicts of Interests: The Authors declare that there are no competing interests.

This article is licensed under a Commons Attribution Non-Commercial 4.0 License

\section{ABSTRACT}

Addison's disease is an endocrine disorder characterized by primary adrenal insufficiency due to various causes. Mycobacterium tuberculosis infection was a major cause in the past but is rare nowadays. We describe a patient admitted to our hospital who was diagnosed with tuberculous Addison's disease.

\section{LEARNING POINTS}

- Tuberculosis is a rare cause of Addison's disease in developed countries and so diagnosis is a clinical challenge and requires a high index of suspicion.

- Tuberculosis should always be considered as a cause of Addison's disease since treating active tuberculosis with steroids alone may have serious consequences.

- Computed tomography findings can be very helpful in the diagnosis of tuberculous Addison's disease.

\section{KEYWORDS}

Addison's disease, Tuberculosis

\section{CASE PRESENTATION}

A 45-year-old Caucasian man was admitted to our hospital because of asthenia, anorexia and weight loss. These symptoms had started approximately 3 months previously and progressively worsening. He also described nausea and epigastric discomfort. In the month before admission the patient was unable to get out of bed to perform basic daily activities.

On examination, the patient was alert and oriented. His temperature was $38.3^{\circ} \mathrm{C}$, blood pressure $75 / 51 \mathrm{mmHg}$, heart rate 95 beats per minute, and respiratory rate 22 breaths per minute. Oxygen saturation was $98 \%$ while breathing ambient air. His lungs were clear to auscultation and the heart sounds were normal. His liver was palpable $2 \mathrm{~cm}$ inferiorly to the costal girdle. He was dehydrated, had generalized cutaneous hyperpigmentation and scattered, brown, macular lesions that were also present on the gums. He had no palpable lymph nodes. The neurological examination was normal.

The patient had previously been healthy and was not taking any medication. He lived in a rural area of northern Portugal and worked as a mechanic. He was married and had two children and a cat. He had not recently travelled.

The results of several examinations preformed before admission were brought by the patient. Blood tests revealed hyponatraemia and hyperkalaemia (131 and $5.8 \mathrm{mEq} / \mathrm{l}$, respectively), and an elevated C-reactive protein and sedimentation rate (105 mg/l and $56 \mathrm{~mm} / \mathrm{h}$, 
respectively). Urinalysis showed leukocyturia (146.6/ $\mu$ l). The patient was HIV negative. The results of electrocardiography, echocardiography and chest radiograph were normal. Upper gastrointestinal endoscopy revealed a sliding hiatal hernia. Abdominal ultrasound showed hepatic steatosis and a thin layer of free fluid in the pelvis. A computed tomography (CT) scan of the chest showed non-specific micronodules throughout the lungs, possible sequelae of lung granulomas. An abdominopelvic CT was also performed and revealed hyperplasia of the adrenal glands with two nodules (one on the right adrenal measuring about $2.2 \mathrm{~cm}$ and one on the left adrenal measuring $1.6 \mathrm{~cm}$ ) (Fig. 1), a thin layer of perihepatic fluid and a greater quantity of peritoneal fluid accumulated in the pelvic cul-de-sac, thickening and densification of the greater omentum and mesenteric fat, and small scattered mesenteric and retroperitoneal lymph nodes. The diagnostic hypotheses suggested by this CT scan were peritoneal dissemination of an occult tumour (peritoneal carcinomatosis) or an inflammatory/infectious process (i.e. peritoneal tuberculosis).
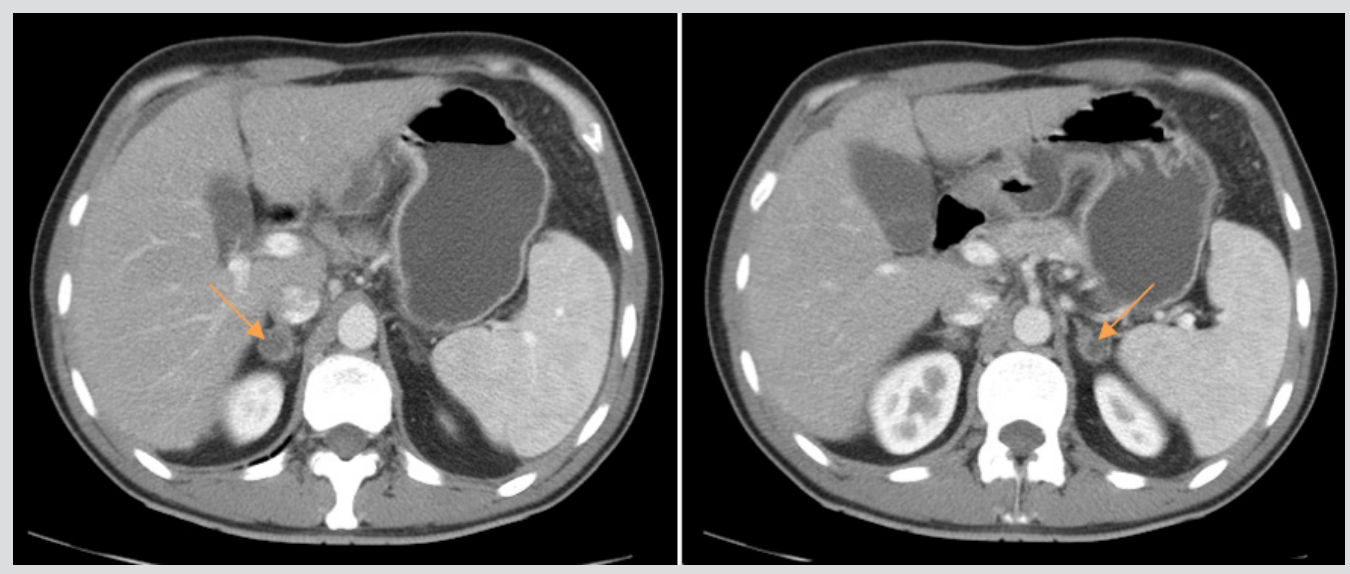

Figure 1. Abdominal CT scan showing bilateral enlargement of the adrenal glands

Methods and Procedures

Based on clinical presentation, there was a strong initial suspicion of adrenal insufficiency. The results of the hormonal study confirmed that suspicion, with a depressed morning plasma cortisol level (1.8 $\mu \mathrm{g} / \mathrm{dl}$; normal range 6.2-19.4 $\mathrm{gg} / \mathrm{dl})$ and an elevated morning adrenocorticotropic hormone (ACTH) ( $>2,000 \mathrm{ng} / \mathrm{l}$; normal value $<63 \mathrm{ng} / \mathrm{l})$. The aldosterone level was also decreased (<2.0 ng/dl; normal range 2-19 $\mathrm{ng} / \mathrm{dl}$ ). A diagnosis of primary adrenal insufficiency was thus established.

In light of the diagnostic hypotheses raised by the CT findings, other diagnostic tests were carried out. The tuberculin skin test (TST) was positive, indicating that the patient had previous contact with Mycobacterium tuberculosis (latent infection or BCG vaccination). A colonoscopy showed no relevant changes. Ultrasound-guided diagnostic paracentesis showed that the peritoneal fluid had some lymphoid and mesothelial cells but no evidence of malignant cells. Bacteriological, mycobacteriological and mycological studies of the peritoneal fluid were negative. Adrenal biopsy was attempted but the amount of tissue collected was insufficient for histological evaluation. Blood, urine and gastric juice were collected for mycobacteriological tests. A strain of M. tuberculosis complex was identified in a urine culture. A diagnosis of tuberculous adrenal insufficiency was thus established.

Treatment was started with intravenous hydrocortisone supplementation and oral fludrocortisone. The patient experienced rapid and marked improvement of his constitutional symptoms as well as resolution of hypotension and analytical changes. Treatment with fourdrug antituberculous therapy (isoniazid, ethambutol, rifampicin and pyrazinamide) was also started. The patient was discharged under oral hydrocortisone and fludrocortisone and antituberculous therapy. He was reassessed 1 month later when he was asymptomatic and had a good general appearance. An abdominopelvic revaluation CT 2 months after the initiation of antituberculous therapy showed a favourable evolution: the adenomegalies and the free fluid in the abdomen had resolved. The patient completed 6 months of antituberculous therapy. The CT scan performed approximately 1 year after finishing this therapy showed disappearance of the left adrenal nodule and a decrease in the size of the right adrenal nodule $(19 \mathrm{~mm})$. Despite good clinical and imaging evolution, the patient has not recovered adrenal function and continues steroid and mineralocorticoid supplementation. He maintains regular follow-up visits to the internal medicine and endocrinology departments. 


\section{DISCUSSION}

Addison's disease is an endocrine disorder characterized by primary adrenal insufficiency and is a relatively rare diagnosis. The disease can occur due to various causes including autoimmune disease, infection (tuberculosis, HIV/AIDS and opportunistic infections), tumour (adrenal metastases and primary adrenal tumours), and vascular and infiltrative disease ${ }^{[1]}$.

In his original paper in 1855, Thomas Addison described the clinical and autopsy findings of 11 patients with adrenal insufficiency, six of whom had disseminated tuberculosis (TB). At that time, TB was the main cause of adrenal insufficiency. The rate of tuberculous adrenal insufficiency has dramatically decreased and autoimmunity is now the primary cause of Addison's disease $(80-90 \%$ of cases) in developed countries $^{[2]}$. However, in developing countries and endemic areas, it continues to account for about 20-30\% of cases of Addison's disease ${ }^{[3]}$. TB as a cause of Addison's disease has become so rare in the developed world that a high index of suspicion is needed to make this diagnosis. However, TB should always be considered as treating active TB with steroids alone can have serious consequences.

CT findings may be very helpful in establishing the diagnosis. Patients with active or recently acquired disease ( $<2$ years) usually have non-calcified bilateral adrenal enlargement, while calcification and atrophy are the norm with more remote infection or inactive disease. Whenever possible, tissue specimens should be obtained for analysis, especially in cases in which adrenal involvement is the only evidence of TB. CT-guided needle aspiration of the adrenal gland has been used successfully to obtain adequate tissue specimens for diagnosis. Adrenal biopsy is not necessary for primary adrenal insufficiency with bilateral adrenal enlargement in a patient with proven extra-adrenal $\mathrm{TB}^{[3]}$.

M. tuberculosis complex spreads to the adrenal glands haematogenously and so is often associated with extra-adrenal infection. The great majority of patients have a previous history of lung and/or pleural infection ${ }^{[4]}$. The most common extra-adrenal manifestations are pulmonary and genitourinary TB. Clinical manifestations may take many years to become apparent and so Addison's disease due to TB manifests relatively late in life, predominantly in individuals aged 40-60 years ${ }^{[4]}$. The possibility of adrenal TB should be considered in cases of fever and enlargement of the adrenal glands, in patients living in areas with a high prevalence of TB, and in patients with a history of TB (either active disease or a positive TST) who present with the classic manifestations of adrenal insufficiency (malaise, anorexia, orthostatic hypotension and skin hyperpigmentation). Laboratory abnormalities may include anaemia, hyponatraemia and hyperkalaemia. Rarely patients may present with acute onset life-threatening adrenal insufficiency ('adrenal crisis') ${ }^{[1]}$.

Treatment recommendations for adrenal TB are similar to those for pulmonary TB. Adrenal enlargement usually improves with antituberculous treatment, while adrenal insufficiency does not ${ }^{[3]}$.

In conclusion, adrenal TB is a rare but important disease entity that must be identified early and treated promptly and aggressively.

\section{REFERENCES}

1. Oelkers W. Adrenal insufficiency. NEJM 1996;335:1206-1212.

2. Nakaoka K, Branch J, Yamaguchi M, Ozeki T, Hazi Y, Tomino T, et al. A case of primary adrenal tuberculosis - a diagnostic quandary. Endocrinol Metab Syndr 2012;1:1-3.

3. Upadhyay J, Sudhindra P, Abraham G, Trivedi N. Tuberculosis of the adrenal gland: a case report and review of the literature of infections of the adrenal gland. Int $J$ Endocrinol 2014;2014:876037.

4. Komura K, Demura H, Saruta T. Addison's disease in Japan: characteristics and changes revealed in a nationwide survey. Intern Med 1994;33:602-606. . 